\title{
Renal Fanconi syndrome with meropenem/amoxicillin-clavulanate during treatment of extensively drug- resistant tuberculosis
}

\author{
To the Editor:
}

We read with interest the papers by TiBER et al. [1,2] describing the effectiveness of meropenem/ clavulanate in treating multidrug-resistant (MDR) and extensively drug-resistant (XDR) tuberculosis (TB) patients. In these analyses, 96 patients were treated with meropenem/clavulanate for a median of 85 (interquartile range (IQR) 49-156) days with six adverse events, and 84 patients were treated with imipenem/clavulanate for a median (IQR) of 187 (60-428) days and three adverse events, none renally related. We report a patient with XDR-TB who developed renal Fanconi syndrome apparently due to meropenem/amoxicillin-clavulanate.

A 25-year-old South Asian female with intermittently treated pulmonary XDR-TB since 2006, presented to the National Institutes of Health (NIH) in 2015 for treatment under an institutional review board-approved clinical protocol. She was started on daily linezolid, bedaquiline, clofazimine and meropenem $1.5 \mathrm{~g}$ i.v. with amoxicillin-clavulanate 500/125 mg orally, both every $8 \mathrm{~h}$. Within $1 \mathrm{month}$, she developed mild hypophosphataemia $\left(1.8-2.0 \mathrm{mg} \cdot \mathrm{dL}^{-1}\right)$. Her renal function and urinalysis remained normal at 3 months. Her sputum cultures converted to negative after 98 days of treatment. At 4 months, she developed a mild normal anion gap metabolic acidosis (bicarbonate $18-19 \mathrm{mmol} \cdot \mathrm{L}^{-1}$ ). After 6-7 months, she developed fatigue, moderate proteinuria $(600 \mathrm{mg} / 24 \mathrm{~h})$, aminoaciduria, glycosuria $(2-3+$ with normal serum glucose), worsening hypophosphataemia with a high urine fractional excretion of phosphorus (24\%) consistent with inappropriate urinary phosphate wasting, and hypouricaemia (uric acid $0.9 \mathrm{mg} \cdot \mathrm{dL}^{-1}$ ), in addition to the normal anion gap metabolic acidosis, all consistent with generalised proximal tubular dysfunction and renal Fanconi syndrome. The aetiology was presumed secondary to medications as the patient did not have other diseases associated with acquired renal Fanconi syndrome. Renal Fanconi syndrome had not been reported with any of her active drugs so, initially, all medications were continued. Renal function remained stable but proteinuria gradually increased to $1200 \mathrm{mg} / 24 \mathrm{~h}$. Urine electrophoresis was consistent with tubular protein losses. Beta-2-microglobulin levels were also elevated, consistent with proximal tubular dysfunction.

Although bedaquiline was considered the most likely culprit due to its limited long-term clinical experience, its 5.5 month half-life made evaluating the effect of stopping this drug challenging. As proteinuria continued to worsen (figure 1) and the patient developed intermittent paresthesias, linezolid was discontinued first. After several days without improvement, linezolid was restarted and bedaquiline discontinued. 1 month later, all TB medications were held due to worsening fatigue and increased urinary protein excretion $\left(2000 \mathrm{mg} / 24 \mathrm{~h}\right.$; eGFR $>100 \mathrm{~mL}$ per min per $\left.1.73 \mathrm{~m}^{2}\right)$. Over the next week, the patient's fatigue, metabolic acidosis, and hypophosphataemia improved and proteinuria declined to $575 \mathrm{mg} / 24 \mathrm{~h}$, despite measurable serum bedaquiline and clofazimine levels. Linezolid was restarted; proteinuria continued to decline and serum phosphorous and bicarbonate returned to baseline. Meropenem/ amoxicillin-clavulanate were added next. Within $48 \mathrm{~h}$, proteinuria and hepatic transaminases increased (figure 1). Meropenem/amoxicillin-clavulanate were again discontinued with improvement in proteinuria and liver function within 3 days.

@ERSpublications

Prolonged use of meropenem/amoxicillin-clavulate to treat XDR-TB may be associated with renal Fanconi syndrome http://ow.ly/dFSD30gCKti

Cite this article as: Abadie ME, Strich JR, Kim T, et al. Renal Fanconi syndrome with meropenem/ amoxicillin-clavulanate during treatment of extensively drug-resistant tuberculosis. Eur Respir J 2017; 50: 1702023 [https://doi.org/10.1183/13993003.02023-2017]. 


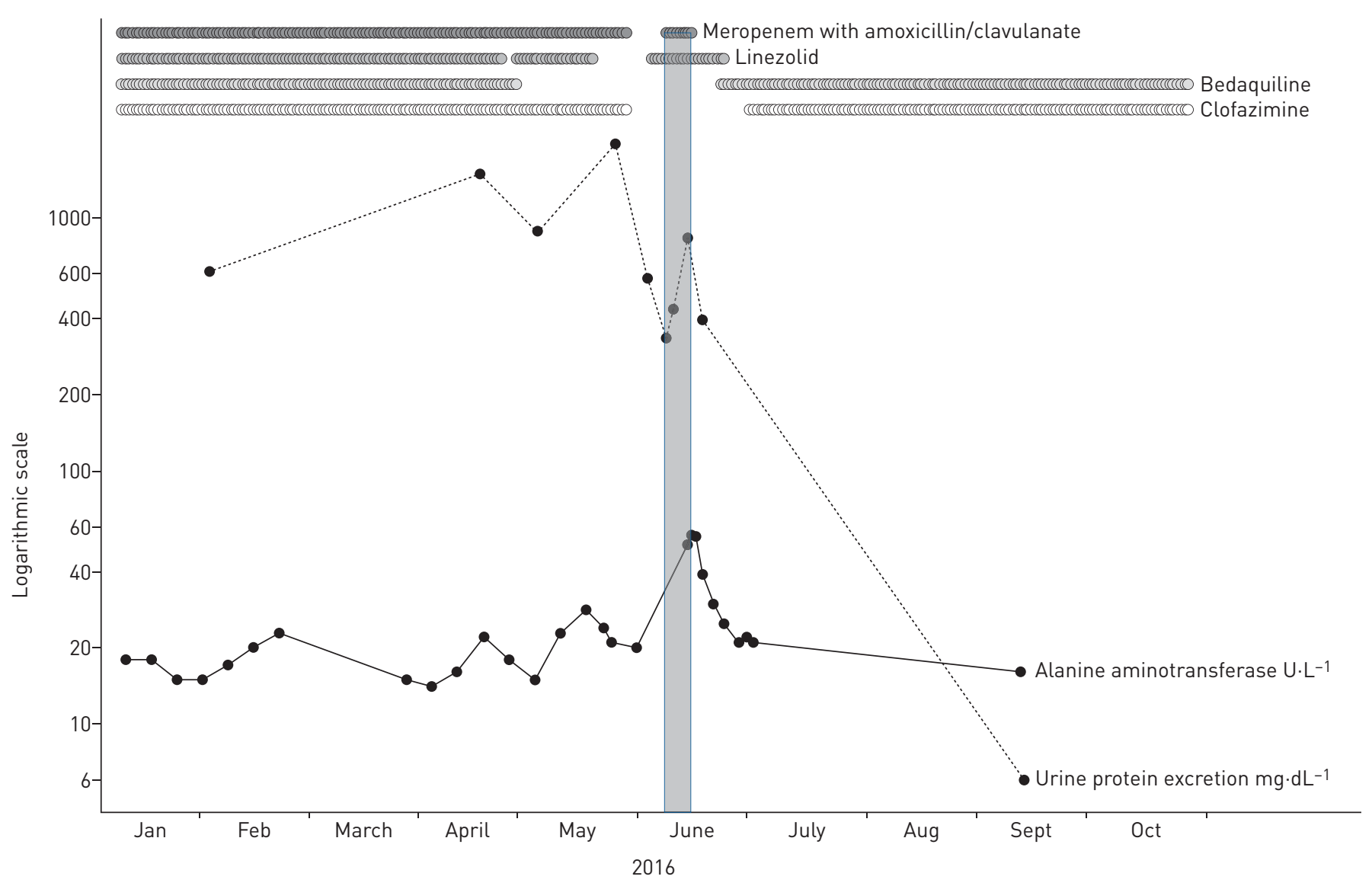

FIGURE 1 Graph showing days on therapy with changes in urine protein excretion and serum alanine aminotransferase over time. Vertical shading indicates the 7 days during which meropenem with amoxicillin-clavulanate was reinitiated. Despite the pause in bedaquiline and clofazimine dosing, serum levels of these drugs remained measurable throughout due to their long half-lives.

Linezolid was subsequently discontinued after 1 year of therapy due to lower extremity neuropathy and weakness; she was maintained on bedaquiline and clofazimine alone. Follow-up urinalysis 3 months after meropenem/amoxicillin-clavulanate were discontinued showed resolution of proteinuria, glycosuria, hypouricemia, hypophosphataemia, and metabolic acidosis. She completed 11 months of meropenem/amoxicillin-clavulanate, 12 months of linezolid, and 27 months of bedaquiline and clofazimine, amounting to 2 years of treatment after sputum culture conversion. Mean QTc interval on treatment was $421.7 \mathrm{~ms}$ (397 $\mathrm{ms}$ on admission). There was no evidence of renal/urinary abnormalities at treatment completion.

Although there are extensive short-term safety data for carbapenems in routine bacterial infections, little long-term data exist. A recent review of carbapenems with clavulanate for MDR/XDR-TB found good efficacy and tolerability without reports of proteinuria or renal adverse events [3]. Renal Fanconi syndrome is characterised by inadequate tubular reabsorption of glucose, bicarbonate, phosphate, uric acid, potassium and amino acids due to dysfunction of the proximal tubule of the nephron, resulting in enhanced urinary excretion. Fanconi syndrome can be due to inherited mutations or acquired from exposure to toxins that injure the proximal tubule (e.g. heavy metals, drugs such as aminoglycosides, tenofovir, cisplatin) or diseases that affect the proximal tubule (e.g. multiple myeloma, amyloidosis, Sjogren syndrome). When caused by a drug, stopping the offending drug usually resolves the disease. Renal Fanconi syndrome has only been attributed to anti-tuberculous treatment twice, both times with rifampin [4, 5]. Drug-induced renal Fanconi syndrome was diagnosed in our patient based on low serum bicarbonate with normal anion gap acidosis (without other identifiable causes such as diarrhoea), normoglycaemic glycosuria, low serum uric acid and phosphorus concentrations with phosphaturia, aminoaciduria and tubular proteinuria. It was temporally associated with meropenem/ amoxicillin-clavulanate. There are currently no other reports of carbapenems, amoxicillin, or clavulanate implicated in renal Fanconi syndrome. The mechanism by which meropenem or amoxicillin-clavulanate may have caused renal Fanconi syndrome is not clear but we believe it is important to raise awareness of 
this potential adverse reaction because of the growing long-term use of carbapenems with amoxicillin-clavulanate to treat drug-resistant TB.

Miriam E. Abadie ${ }^{1}$, Jeffrey R. Strich ${ }^{2}$, Tiffany Kim ${ }^{3,4}$, Yingda L. Xie ${ }^{5}$, Kevin P. Fennelly ${ }^{6}$, Kenneth N. Olivier ${ }^{6}$, Meryl Waldman ${ }^{7}$, Kriti Arora ${ }^{5}$, Steven M. Holland ${ }^{5}$ and Ray Y. Chen ${ }^{5}$

${ }^{1}$ Dept of Medicine, Georgetown University Hospital, Washington, DC, USA. ${ }^{2}$ Division of Clinical Research, National Institute of Allergy and Infectious Diseases, National Institutes of Health, Bethesda, MD, USA. ${ }^{3}$ Dept of Pharmacy, Clinical Center, National Institutes of Health, Bethesda, MD, USA. ${ }^{4}$ Division of Pharmacovigilance, Office of Surveillance and Epidemiology, Center for Drug Evaluation and Research, Food and Drug Administration, Silver Spring, MD, USA. ${ }^{5}$ Division of Intramural Research, National Institute of Allergy and Infectious Disease, National Institutes of Health, Bethesda, MD, USA. ${ }^{6}$ Pulmonary Branch, Division of Intramural Research, National Heart, Lung, and Blood Institute, National Institutes of Health, Bethesda, MD, USA. ${ }^{7}$ National Institute of Diabetes and Digestive and Kidney Diseases, National Institutes of Health, Bethesda, MD, USA.

Correspondence: Ray Y. Chen, 9000 Rockville Pike, Bldg 33, Rm 2W20, Bethesda, MD 20892, USA.

E-mail: ray.chen@nih.gov

Received: Sept 292017 | Accepted after revision: Oct 122017

Support statement: This work was supported by the intramural research programs of the National Institute of Allergy and Infectious Diseases, National Heart, Lung, and Blood Institute, and the National Institute of Diabetes and Digestive and Kidney Diseases, all at the National Institutes of Health. Funding information for this article has been deposited with the Crossref Funder Registry.

Conflict of interest: Disclosures can be found alongside this article at erj.ersjournals.com

\section{References}

1 Tiberi S, Payen MC, Sotgiu G, et al. Effectiveness and safety of meropenem/clavulanate-containing regimens in the treatment of MDR- and XDR-TB. Eur Respir J 2016; 47: 1235-1243.

2 Tiberi S, Sotgiu G, D'Ambrosio L, et al. Comparison of effectiveness and safety of imipenem/clavulanate- versus meropenem/clavulanate-containing regimens in the treatment of MDR- and XDR-TB. Eur Respir J 2016; 47: $1758-1766$.

3 Sotgiu G, D'Ambrosio L, Centis R, et al. Carbapenems to treat multidrug and extensively drug-resistant tuberculosis: a systematic review. Int J Mol Sci 2016; 17: 373.

4 Min HK, Kim EO, Lee SJ, et al. Rifampin-associated tubulointersititial nephritis and Fanconi syndrome presenting as hypokalemic paralysis. BMC Nephrol 2013; 14: 13.

5 Park JT, Lee S, Kim W, et al. A case of acute tubulointerstitial nephritis associated with rifampin therapy presenting as Fanconi-like syndrome. Chonnam Med J 2017; 53: 81-82.

The content of this work is not subject to copyright. Design and branding are copyright ()ERS 2017. 